\title{
Two-dimensional and dynamic (2DD) method of visualization of the flow characteristics in a convection boundary layer using infrared thermography
}

\author{
Jacek A. Patorski ${ }^{{ }^{*}}$, Günter S. Bauer ${ }^{a}$ and Sergei Dementjev ${ }^{b}$ \\ ${ }^{a}$ Paul Scherrer Institute, Spallation Neutron Source Division, CH-5232 Villigen-PSI, Switzerland \\ ${ }^{\mathrm{b}}$ University of Latvia, Institute for Physics, LV-2169 Salaspils-1, Latvia
}

\begin{abstract}
The paper presents the two-dimensional and dynamic (2DD) method of using infrared (IR) thermography for the visualization of the cooling efficiency of a heated wall, as this method was applied in an experimental investigation. The 2DD method allows the outer surface temperature measured by the IR thermography device to be worked out relative to the bulk coolant-fluid temperature. In this way the 2DD method makes visible the qualitative and quantitative flow characteristics within the thin contact layer at the inner surface of the wall. This flow characteristics, and more specifically the pattern of stream lines (for the detection of dead zones) and the distribution of the temperature differences between the temperature on the window outer surface and the bulk temperature of the coolant, determine the cooling efficiency. Finally animated IR thermogram sequences could be generated, allowing the spatial and temporal behavior of the flow/cooling behind the wall to be observe (for example see internet page http://wwwl.psi.ch/www_gfa_hn/asq/projects/liquid/liquid.html).

The study of the cooling of the proton beam entry window described in this paper is part of the development program of neutron spallation sources with liquid metal targets ${ }^{1 .}$. The basic idea of the experiments was to use the real interacting materials (mercury as liquid metal and steel as window's wall) and observe the cooling effects of the mercury flow on the heated wall of the hemispherical shell of the mockup window. Different geometrical configurations of the inner flow guide tubes of the flow and different pumping velocities were examined in view of finding the optimal cooling solution.
\end{abstract}

Keywords: IR-thermography measurement, visualization of internal flow within convection boundary layer, heat transfer visualization.

\section{INTRODUCTION}

As part of the development program of the neutron spallation sources with liquid metal targets ${ }^{1 .)}$ p.106-110, the cooling of the proton beam entry window was experimentally investigated at the Paul Scherrer Institute (PSI) and at the Institute of Physics of the University of Latvia (IPUL) in cooperation with European Spallation Source (ESS) project. PSI has built and since 1996 operates the Spallation Neutron Source (SINQ) with solid targets, but prospectively a liquid metal target as neutron source seems to be more efficient.

For the security of the future ESS/SINQ liquid metal targets, the integrity of the proton beam entry window must be guaranteed for the planned lifetime of the targets. In this respect, one of the most important tasks during the operation of the ESS/SINQ facilities will be to keep the temperature of the target window within the prescribed temperature range. In reality the density of power deposition of a proton beam in the window, e.g. at SINQ, will amount to ca. $70 \mathrm{Watt} / \mathrm{cm}^{2}$. Such power density causes strong heating of the window. Therefore, to avoid instantaneous local overheating (by hundreds of degrees centigrade) and to guarantee the integrity of the window, the cooling of the window by the liquid metal itself, flowing inside the target-shell, must be proven to be reliable and sufficient.

The main goal was to study experimentally questions of heat transfer between the steel wall of the hemispherical window shell and the fluid mercury and to observe related flow distributions in various geometrical configurations. Figure 1 shows examples of two such configurations with different inner guiding tubes. The experiments were carried out at IPUL in Riga-Salaspils in Latvia, using a test facility loop with a capacity of about 6 tons of mercury. The first phase of the experiments, which used the target shell geometry of SINQ and mercury as liquid metal is now completed.

\footnotetext{
* Correspondence: Email: jacek.patorski@psi.ch ; Internet page : http://wwwl.psi.ch/www_gfa_hn/asq/asqhome.html ;
} Telephone: +41563102660, Fax: +41563103131 

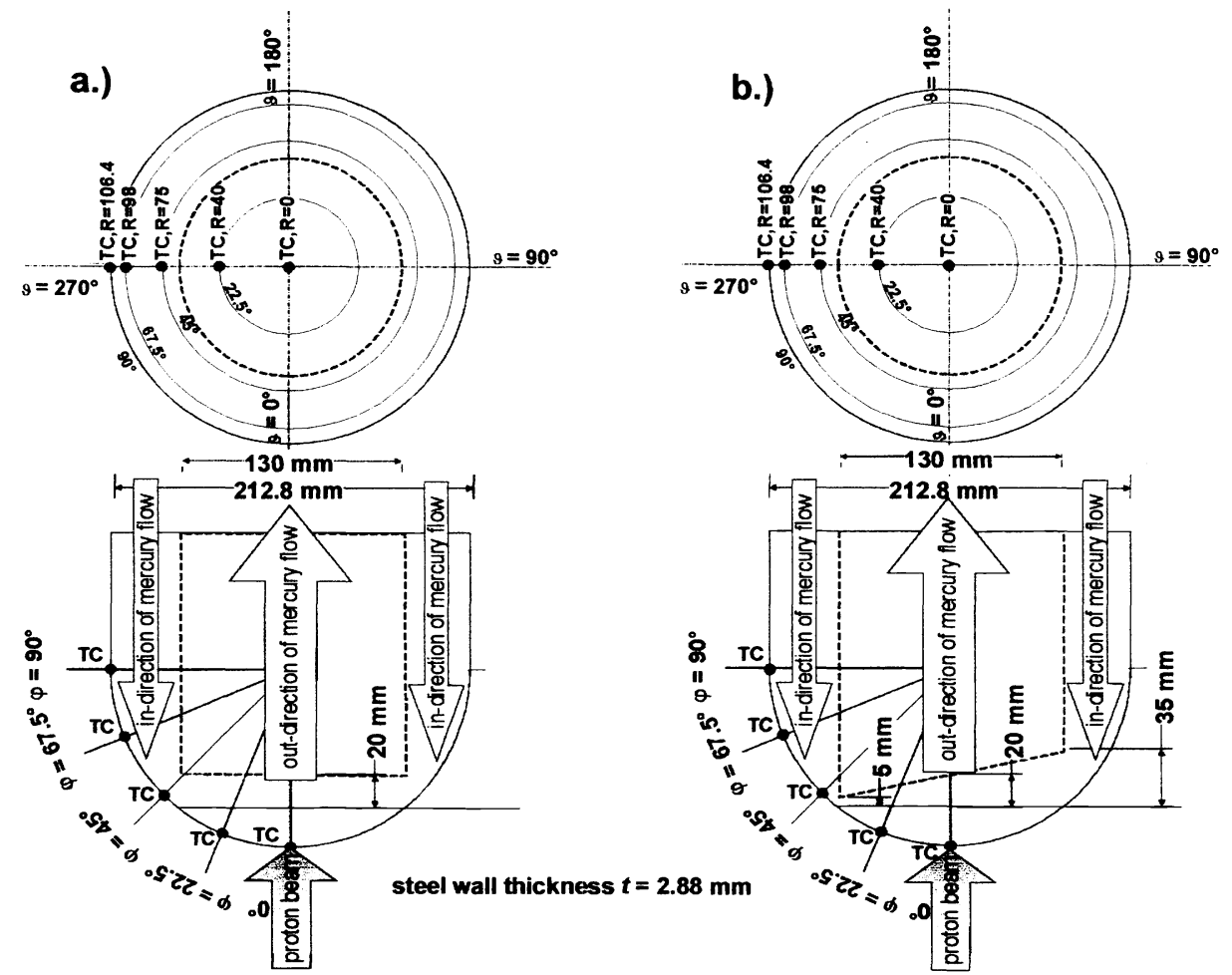

Figure 1. Geometry of the hemispherical entrance window of the SINQ target, showing the internal guide pipes, directions of mercury flow, direction of the proton beam impact and the positions of the thermocouples (TC). (TC are in pairs on the inner and outer surfaces of the wall), a.) flat configuration and gap of $2 \mathrm{~cm}$, b.) skew configuration and gap of $2 \mathrm{~cm}$.

The methods of investigation developed and used in three groups of separate experiments include:

- Ultrasonic Velocity Probes (UVP), based on a through-the-wall measurement of the Doppler effect in the fluid ${ }^{2 .)}$, for the investigation of the $3 \mathrm{D}$ velocity distribution inside the target shell,

- Heat Emitting Temperature Sensitive Surfaces (HETSS) ${ }^{3 .)}$, for the measurement of the convection heat transfer coefficient on a coarse mesh and

- surface IR thermography ${ }^{4 .)}$, for the non-contact temperature measurement on a fine mesh, in order to find out the time/space behavior of the cooling efficiency of the window wall.

The basic idea of the IR thermography experiments was to observe the cooling effect of the mercury flow (serving as sole coolant) on the heated wall of the mockup window. The mercury flow was induced either solely through buoyancy effects, or through these effects supplemented with pumping. For these experiments a mockup of the hemispherical target window of steel was used. The geometry adopted was that of SINQ i.e. window diameter was $212.8 \mathrm{~mm}$ and window wall thickness $2.88 \mathrm{~mm}$. Figure 1 shows schematically the geometry of the target, the position of the proton beam, the directions of the mercury flow and the positions of the thermocouples (TC), which have been used for control/calibration tests.

Data from the IR thermography experiments, which have been measured with high time/space resolution $(20 \mathrm{~Hz} / 1.25 \mathrm{mrad})$ and high sensivity $\left(0.1^{\circ} \mathrm{C}\right)^{5 .)}$, are a base for the 2DD method presented in this paper.

\section{GOALS OF THE IR THERMOGRAPHY INVESTIGATIONS OF THE WINDOW COOLING}

The goals of the IR thermography experiments presented here are of both quantitative and qualitative nature.

\#1. To learn about the phenomenology of the cooling of the liquid target with the help of IR-thermography (To what extent can IR thermography help to make the internal mercury flow visible ?). The dynamical observation of the change of the temperature pattern on the outside surface of the mockup window, for constant heating and variable mercury flow conditions, was planned.

\#2. With the help of IR thermography and thermocouples, and for constant heating and variable mercury flow conditions, to measure the absolute temperature of a mockup target window made of a material suited for the real use and to measure simultaneously the absolute mercury bulk temperature. Then, from the set of experimental results, to perform : 
- a numerical evaluation and visualization of the effectiveness of different cooling parameters (i.e. relative comparison of cooling efficiencies between different geometrical configurations of inner guiding tubes and different pumping flow velocities),

- a calculation and visualization of the local thermal resistivity (a reciprocal of the local convection heat transfer coefficient) and a comparison with the values obtained by the HETSS investigations ${ }^{3 .)}$ for all tested cooling configurations.

\section{THE MAIN IDEA OF THE 2DD METHOD}

Any modern IR thermography device has a two-dimensional and dynamic (2DD) capability, but simply observing and recording thermograms of a wall that is simultaneously heated and cooled by some method will not allow us to reach the two goals we have outlined in section 2. The essential content of the 2DD method presented here (besides the use of thermography equipment) lies in answering the following questions :

- How must the wall be heated ? What must be measured, and how ? How must the measured data be processed ?

These questions can be answered by applying an algorithmic procedure, which we will present in section 4 and by describing how it was applied in a particular situation. This presentation illustrates both the theoretical and the practical aspects of the method. The main points of the concept used to visualize the pattern of stream lines and the distribution of local thermal resistivity is outlined below. When a flow occurs inside a pipe, it is known that small "micro" changes in the velocity and "macro" dislocations in the pattern of stream lines (ie the development of vortexes) appear in the boundary layer. Consequently the local convection coefficient will differ slightly in time and in space and the temperature on the inner surface will also change a little. If these "micro" temperature changes at the inner surface can be inferred from the IR thermography measurements of the outer surface, we will be able to recognize the pattern of stream lines from the changes in the pattern of the temperature field, and this will allow us to reach our first goal.

If not only the absolute temperature of the bulk fluid, but also the distribution of the absolute temperature field over the inner surface could be known (just as the absolute temperature field over the outer surface is known from the IR thermography measurements), and if moreover the local heat flux distribution in the heated wall of the mockup were known, then we would be able to to estimate the local thermal resistivity field, and so reach also our second goal.

Because cooling takes place in the convection boundary layer on the invisible inner surface of the window shell, and because IR thermography can measure only the outer surface of the shell, we have to consider the conditions for the "micro" transient conduction phenomena which are responsible for the heat flux through the wall. This consideration must take into account and also places conditions on the type of wall heating which was chosen for the IR thermography.

A first condition for the wall heating is that it should come from inside, because heating from outside will be partially reflected at the wall surface and so perturb the IR thermography measurement; a second condition is that the heat flux be known, so that the local thermal resistivity can be evaluated. We have decided to use so-called "skin effect" heating (SEH) for volumetric heat generation; for more details about SEH see later subsections 4.1 and 4.2.

FEM calculations of the "micro" transient conduction problem" have shown that with our experimental conditions (i.e. the type of steel used in the window, the thickness of the window wall and the range of heat fluxes achieved with SEH heating and with the bulk temperature of the mercury as coolant) the "micro" changes of temperature will be propagated to the outside surface with very little delay and can be measured with IR thermography equipment ${ }^{5 .)}$ with sufficient accuracy.

Because the "skin effect" heating was the most characteristic feature of the whole series of experiments done with IR thermography, we have named them the SEH experiments.

\subsection{Set-up of SEH-experiments}

\section{SEH-EXPERIMENTS}

With respect to goal \#1, the expectation that IR-thermography can open new insights into the phenomenology of the cooling process of the target window played a large role in the planning of the SEH-experiment. The entire set-up with all the instrumentation of the SEH-experiment is shown in Figure 2. The SINQ target window mockup has been fabricated from stainless steel DIN 1.4057 (AISI 431) and placed in the test section of the mercury loop in the distance of $1900 \mathrm{~mm}$ over the position of the IR thermography scanner. The space between the mockup and the IR scanner has been screened against reflections with massive walls of the pit below the loop and the material curtain. Some additional details of the mockup preparation are shown and described in Figure 3.

\footnotetext{
*A presentation of this calculation is foreseen in another publication.
} 


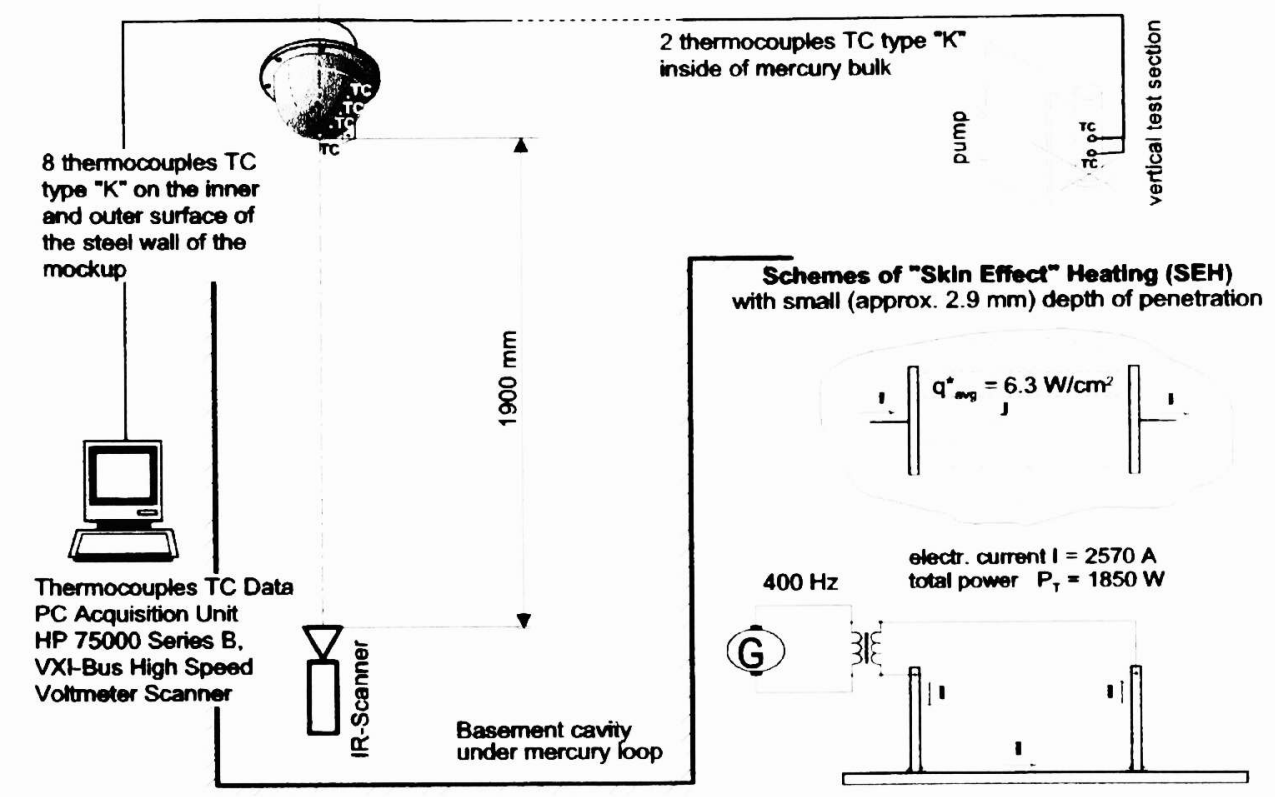

Figure 2. Set-up of SEH-experiment. Schemes of the loop, of the used instrumentation and of the electric SEH heating.
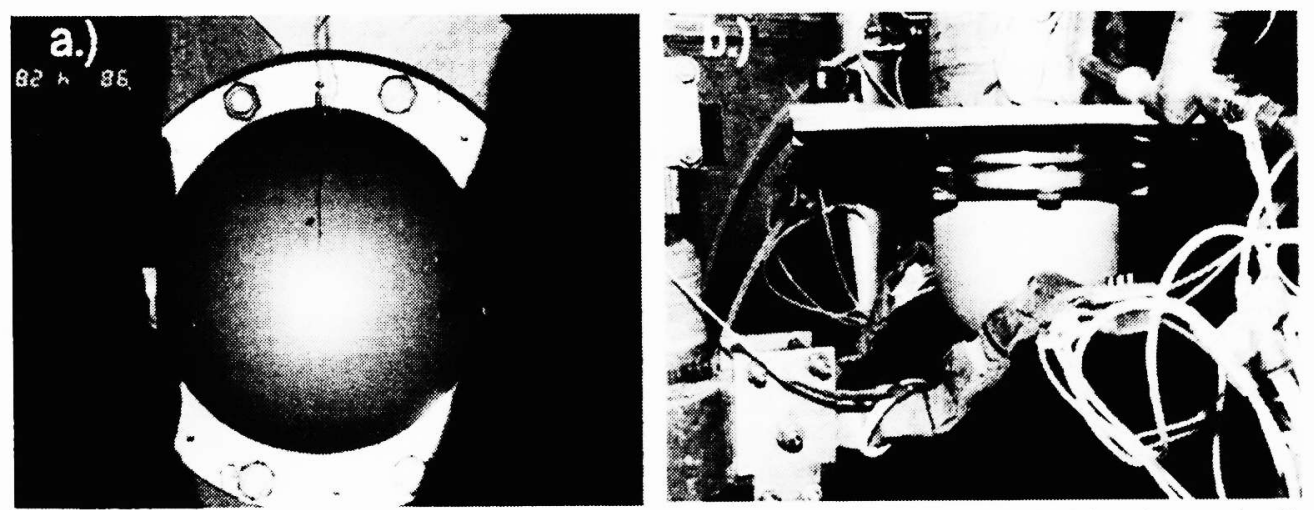

Figure 3. a.) The sandblasted $\left(R_{a}=3.2 \mu \mathrm{m}, \mathrm{N} 8\right)$ surface of the steel mockup, surrounded by the antireflection black curtain and showing the soldered copper electrodes of the SEH-heating on the horizontal axis and the thermocouple wires on the vertical axis. b.) The mockup mounted to the lower end of the mercury loop. The components of the SEH-heating: buses to the transformer and the water cooling plastic tubes are connected to both electrodes.

A suitable heating mechanism for the window was chosen in view of optimizing the conditions for IR-thermography measurements. Thus the following measures and design choices were taken:

-To heat the target window by volume heating (the electric "Skin Effect" Heating SEH) inside the thickness of the window wall. Volume heating is well-suited for use with IR thermography because heat reflection from the window surface is avoided. In the converse case of surface heating with an outside heat source (like, for example, a hot-air ventilator or a powerful light projector), the non-blackbody surface of the window reflects the infalling heat and disturbs the IR thermography measurements.

- To screen the mockup with a surrounding anti-reflection black curtain (see Figure 3.a) in order to avoid the effects of ambient heat sources at the sides (e.g. electric instruments/components or walking persons).

- To enhance the emissivity of the window surface and make it uniform. To this end the outer surface was sandblasted to the roughness $R_{a}=3.2 \mu \mathrm{m}$ or roughness class $N 8$ (as per DIN 4768 and ISO 1302).

-To perform an exact calibration of the IR thermography equipment after each change of experimental configurations The so-called emissivity chart technique was used, which gives an individual value to the emissivity $\varepsilon$ of each measurement pixel. 


\subsection{The Skin Effect Heating}

By design, so-called "Skin Effect" Heating, well-known from the theory of electric induction, was chosen as most appropriate for this experiment and the transformer was connected directly to the mockup through copper electrodes soldered to the window. The main parameters of the heating scheme are shown in the lower right part of Figure 2. Some components of the real SEH-heating can be seen in Figure 3. In this type of heating, increasing the frequency of the current passing through a bulk conductor will cause energy to be deposited in a thin sheet near the outer surface of the conductor. The depth of penetration $\delta$ of this layer is given by equation (1) and decreases with increasing frequency. In our case, the parameters of the equation were chosen so that the depth of penetration should be less than the window thickness $t=2.88 \mathrm{~mm}$, in order to avoid heating the mercury. In addition the constraints given by the existing generator and transformer at IPUL in Riga ${ }^{6 .)}$ led to the choice of the frequency $f=400 \mathrm{~Hz}$.

$$
\delta=\sqrt{\frac{2}{\sigma \cdot \mu_{r} \cdot \mu_{0} \cdot \omega}}=2.86 \mathrm{~mm}
$$

where $\delta$ is depth of penetration, $\sigma$ - electric conductivity, $\mu_{r}$ - relative magnetic permeability, $\mu_{0}$ - magnetic field constant; $\mu_{0}=4 \Pi 10^{-7}[\mathrm{Vs} / \mathrm{Am}], \omega$ - angular velocity; $\omega=2 \Pi \cdot f$ where $f$ - AC frequency.

Because of the magnetic characteristics, the mockup was made of martensitic chromium-nickel stainless steel DIN 1.4057 (AISI 431) specially for these tests. For the adopted arrangement of the electrodes (distance of approximately 180 $\mathrm{mm}$ ) and the heating current of $I=2570 \mathrm{~A}$ used for the experiment, the relative permeability of steel DIN 1.4057 is $\mu_{r}=54$. Also the electrical resistivity of this steel at $20^{\circ} \mathrm{C}$ is $\rho=0.7 \Omega \mathrm{sm}^{2} / \mathrm{m}$, corresponding to an electric conductivity, as used in Equation (1) of $\sigma=1^{\prime} 428^{\prime} 571(\Omega \mathrm{m})^{-1}$. The reliability of SEH-heating was enhanced by imposing a limit to the value of the current $(I=2570 \mathrm{~A})$ and to the duration of the heating ( 5 minutes). In addition a very efficient water cooling of the electrodes was installed, in order to avoid transfer of heat from the buses to the mockup, see Figure 3.b. The SEH used produces a power deposition density within the range of 2 to $11 \mathrm{~W} / \mathrm{cm}^{2}$ in the central region of the window. This value is smaller than the $70 \mathrm{~W} / \mathrm{cm}^{2}$ deposed in the real case through the proton beam, but is enough to study the characteristic of the target self-cooling process. The average power density $q^{*}$ used in the experiments was $6.3 \mathrm{~W} / \mathrm{cm}^{2}$ and the total power rate was approx. $Q=1850 \mathrm{~W}$.

\subsection{Instrumentation of SEH-experiment}

As shown in Figure 2, the general instrumentation used in these experiments consists of two temperature measurement devices: the IR-thermography equipment and the thermocouples scanning equipment. In addition, simple instruments were used to measure the humidity and the atmospheric and ambient temperatures.

For the calibration pre-tests, which were powered with the $1000 \mathrm{~W}$ heater (see the description in subsection 4.4. for more details), a typical standard power control equipment with its own steering and security thermocouples was used.

The precise control, needed to keep the pumping parameters of the loop at their prescribed values, to hold the SEHheating constant and to maintain the security of the whole facility, was assured by equipment belonging to IPUL, and which will not be described in this paper.

The IR thermography equipment of PSI (FLIR-AGEMA THV900 SWTE) is a non-contact measurement system, which allows $2 \mathrm{D}$ thermograms in the infrared short wave spectrum range $(2-5.6 \mu \mathrm{m})$ to be registered. The IR-thermograms can be collected as single frames or as sequences. The time resolution for on-line recording by the IR-scanner and controller vary from $20 \mathrm{~Hz}$ for frames consisting of 128 lines at 204 pixels up to $3500 \mathrm{~Hz}$ for single lines of 204 pixels. The spatial resolution depends on the optic lens of the IR-scanner. In the SEH experiments a $10^{\circ}$-teleobjective lens with a spatial resolution of $1.25 \mathrm{mrad}$ was used. For a more detailed technical specification of FLIR-AGEMA THV900 SWTE, see User's Manual ${ }^{5 .)}$.

The data acquisition system for the thermocouples consists of a Hewlett Packard high-speed digital voltmeter scanner model HP 75000 series B, based on the VXI bus architecture and including a mainframe HP model E1300A, a measurement slot module HPE1313A as 32-channel scanning A/D and a PC running the HP VEE software package in the MS Windows environment. Very thin thermocouples (diameter $0.25 \mathrm{~mm}$ ) of the "K" type, produced and calibrated at PSI by the group "Sensorik und Sonderverfahren" guaranteed response times shorter than $20 \mathrm{~ms}$ and reached the high accuracy of $+/-0.2^{\circ} \mathrm{C}$.

The data sets from the IR thermography and from the TC measurement must be recorded simultaneously. In order to achieve this synchronization, a special trigger unit was built and used to start each experiment. 


\subsection{IR Calibration Pre-Tests}

The preparations described below are a part of the intense efforts which were made to measure the absolute temperature

$\boldsymbol{T}_{\text {o.wall }}$ on the outer surface of the mockup. The difference to the absolute value $T_{\text {bulk }}$ of the mercury bulk temperature is essential for the evaluation of the results (see also equations (10) and equation (13)).

The use of IR-thermography to derive absolute values of temperature requires the value of the emissivity $\varepsilon$, a radiative property of the surface, to be known. The emissive power $\boldsymbol{E}$ of the surface, which is the quantity directly measured in the IR-scanning, is less than the corresponding quantity $E_{b h}$ of blackbody radiation at the same temperature and is given by the Stefan-Boltzmann law equation (2)

$$
E=\varepsilon \cdot \sigma \cdot T^{4}
$$

where $\varepsilon$ is emissivity; radiative property of the surface, values in the range $(0-l), \sigma$ - Stefan-Boltzman constant; $\left(\sigma=5.67 \mathrm{E}-8 \mathrm{~W} / \mathrm{m}^{2} \mathrm{~K}^{+}\right), T$ - absolute temperature in $K$.

For each measurement pixel, the emissivity can be expressed as a function, equation (3) of the spectral range $\lambda$, the temperature $T$, the direction of radiation $\phi$ (i.e. the angle between the view line and the normal to the radiating surface) and the roughness $R_{a}$.

$$
\varepsilon=F\left(\lambda, T, \phi, R_{a}\right)
$$

Because the spectral $\lambda$-dependency of the IR scanner-detector is generally constant within the spectral range used (in our case $\lambda=2-5.6 \mu \mathrm{m}$ ), only the influence of the remaining three variables has to be considered for our experiments. These are: the cleanliness/roughness $\left(R_{a}\right)$ of the surface, the position (i.e. the direction view angle $\left.\phi\right)$ of the hemisphere relative to the IR scanner, and the temperature of the window surface $(T)$. In order to find these dependencies, special calibration pre-tests were planned and performed at PSI and again "in situ" on the experiment stand at IPUL. The calibration pre-tests also served to estimate spectral range directional emissivity and to verify that the IR measurements were accurate and reproducible.

The aim of the calibration test was to heat the outer surface of the mockup to a stable and uniform temperature and to record this temperature with IR thermography and TC equipment, using the TC values as reference for the IR thermography. For this, we used an electronically controlled electric heater (power $1000 \mathrm{~W}$ ) to heat just enough mercury to fill the mockup (approx. 7 litres). Good mixing of the mercury was achieved with gas bubbles. In this way, a uniform temperature field was established on the whole hemispherical surface of the mockup and the reference IR- and TC-measurements were done from precisely the same position as later in the main experiment. At the start of each calibration test, several steps of temperature within the range from $15^{\circ} \mathrm{C}$ up to $70^{\circ} \mathrm{C}$ were measured in order to obtain the temperature dependence of the emissivity.

After that, when the steady state for the desired temperature level had been reached, the simultaneous recording of the IR thermography sequence and the TC-measurement was started for a few minutes. The position of the ten TC used to measure the reference temperature and to control the uniformity of the temperature field is shown in Figure 1.

The results of the calibration pre-tests are stored in so-called emissivity charts; for each experiment configuration, charts for several temperature levels were stored. Figure 4.a shows a typical spectral range directional emissivity chart and Figure 4.b the distribution profile of this emissivity along the diameter LI01.
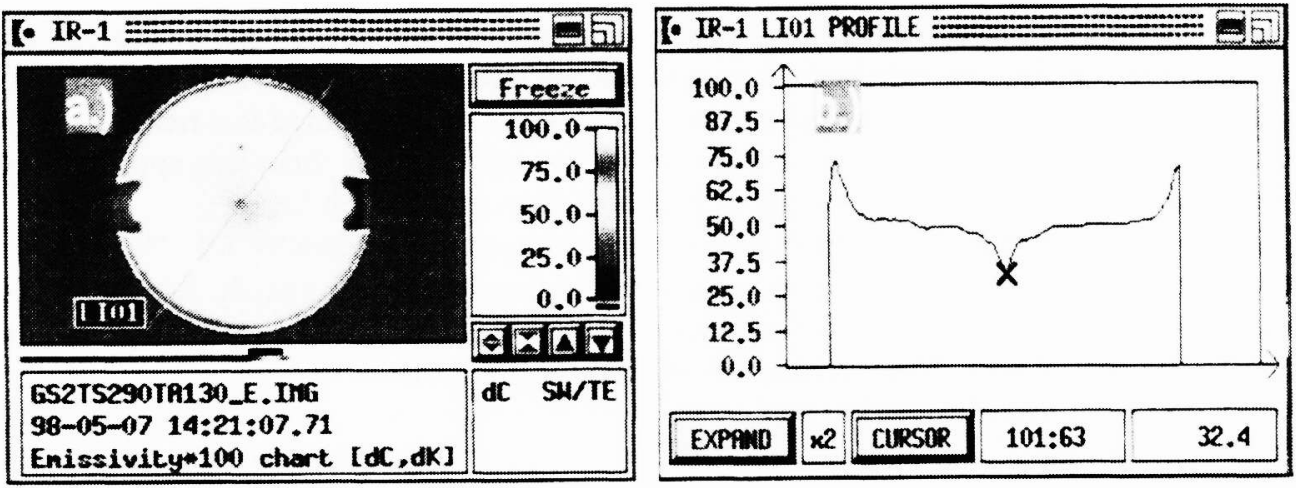

Figure 4.a.) Emissivity chart for a uniform temperature field of $29^{\circ} \mathrm{C}$ and for an experiment with skew gap of $2 \mathrm{~cm}$. b.) Emissivity profile along the diameter LI01 obtained from the chart of Figure 4a. 
The direction view angle $\phi$ has the greatest influence on emissivity: the spectral emissivity of the hemispherical mockup steel surface in axial view varies from $\varepsilon=0.35$ in the central region; $\phi=2^{\circ}-10^{\circ}$, to $\varepsilon=0.74$ on the edge; $\phi=85^{\circ}$ (see Figure 2 for the IR scanner position). The directional $(\phi)$ variation of emissivity is as expected for metals ${ }^{7 .)}$, lower in the middle and quite constant in the area for $\phi=10^{\circ}-40^{\circ}$, and for the area from $\phi=40^{\circ}-85^{\circ}$ rises faster. The lowest value of $\varepsilon=0.324$, measured in the exact middle of the profile on Figure 4.b, is caused by the polished metal of the small plate which holds the thermocouple in place. Small values of $\varepsilon$ were also obtained for the thermocouple wires and for the polished copper electrodes, visible on Figure 4.a. The emissivity charts are the basis for the corrections to the raw IRthermogram sequences and are extensively used during many data evaluation steps.

\subsection{Realization and Progression of the SEH measurements}

In general a similar procedure was followed for all configurations of the SEH experiments.

1. The inner guide pipe and mockup were mounted to the loop and the guide pipe gap was set.

2. Calibration pre-tests were performed for a few temperature levels in the range expected on the surface during the SEH experiments. This was necessary because after each change of the gap the surface cleanness and the position of the mockup are slightly different. The calibration process was time-consuming because the loop had to be opened from the top in order to install the $1000 \mathrm{~W}$ heater, the mixing procedure could only be performed through the drain nozzle and the desired temperature had to be reached slowly.

3. The calibration equipment was removed and the loop was closed and filled with mercury. For the measurement of the mercury bulk temperature two thermocouples were displaced from the edge of the mock-up to the mercury bulk inside the loop. Then a series of experiments with different pump flow rates and different heating were done. For all experiments with forced mercury flow the pump and the water-cooling of the electrodes had to be started first.

4. After that the joint IR-thermography and thermocouple measurements were simultaneously started with the triggering device. Finally, after approximately 30 seconds, the SEH heating $(2570 \mathrm{~A}$ at $400 \mathrm{~Hz} \mathrm{AC})$ was switched on for 4 to 5 minutes. The measurements continued for a few minutes after each switch off of the heating and the end of the datataking terminated the experiment.

\subsection{Theoretical model for evaluation of the data measured in SEH-experiments}

Because IR-thermography can give only the values of the temperature $T_{\text {o.wall }}$ of the outer surface, the problem arises of estimating the difference between this temperature and the temperature $T_{i, w a l l}$ at the inner surface of the window. This aspect is discussed below. Knowledge of the behaviour of $\Delta T_{\text {wall }}=T_{\text {owall }}-T_{\text {i.wall }}$ during the SEH experiments is essential for the interpretation of the IR-thermography measurements.

As mentioned in subsection 4.1, the mockup was surrounded by an anti-reflection curtain of black material. The temperature $T_{a m b}$ of the curtain was controlled during the measurements; it remained equal to the air temperature and varied between 12 and $20^{\circ} \mathrm{C}$. With the exception of the neighborhood of the electrodes, the absolute temperature $T_{\text {ow wall }}$ of the outer surface of the mock-up was in general lower or approximately equal to $T_{a m b}$ during all the experiments. The absolute ambiant temperature was low and the difference between the ambient temperature $T_{a m b}$ and $T_{\text {o.wall }}$ was small, so that the energy radiation flux $q^{*}$ rad can be neglected, especially in comparison with the convective heat flux $q_{\text {conv }}^{*}$ on the inner surface. A numerical example in the report ${ }^{4 .)}$ has shown, that flux ratio $q^{*}{ }_{r a d} / q^{*}{ }_{\text {conv }}$ is in the worst case smaller than $10^{-3}$ and the influence of the radiative energy exchange on the outer surface of the mockup can be neglected.

Above the mockup, the curtain was tightly fixed to a round wooden plate supported on the flange of the mockup (see Figure 3.a) so as to impede effectively any air movement. It therefore can be assumed that neither significant radiation nor free air convection took place on the outer surface and that the average heat flux there was approximately zero. In conclusion, adiabatic boundary conditions can be assumed on the outer surface of the mockup.

As the wall thickness $\delta=2.88 \mathrm{~mm}$ of the mockup is a small fraction of its diameter $D=212.8 \mathrm{~mm}$, allows the considerations to be made in the context of the 1-D steady-state conduction model. This model can be applied to a small local area of the mockup wall at a distance from the electrodes sufficient to guarantee that the predominant heat flux is normal to the surface, e.g. in the central part. The main aspects of the model are shown in Figure 5. As was mentioned in subsection 4.2 , the conversion from electrical to thermal energy will take place only within the thickness of the wall and at a local volumetric rate denoted by $q_{v o l}$. The consequence of this heat generation is that the heat flux is no longer independent of $x$, and this must be considered in the model calculations.

The small ratio of the thickness $\delta$ to the diameter $D$ of the hemispherical mockup and as inward convective heat flux perpendicular to the wall predominates, the simple steady-state 1-D model Figure 5 with uniform energy generation per unit volume $q_{v o l}=$ constant and constant thermal conductivity $k$, can be used for the plane wall. 


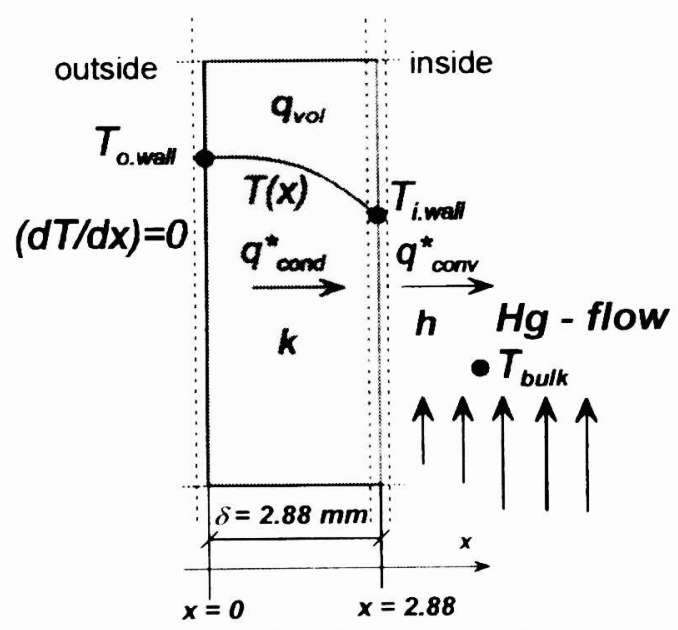

Figure 5. 1-D steady-state model for conduction of uniformly-generated heat, as applied to the SEH experiments.

The appropriate heat equation (4), is then

$$
\frac{d^{2} T(x)}{d x}+\frac{q_{v o l}}{k}=0
$$

where $k$ is thermal conductivity, $q_{v o l}$ - local energy generation per unit volume.

The general solution of equation (4) is

$$
T(x)=-\frac{q_{\text {vol }}}{2 k} \cdot x^{2}+C_{1} \cdot x+C_{2}
$$

The constant of integration $C_{1}=0$ results directly from the assumption of adiabatic boundary conditions $(d T / d x)_{(x}(0)=0$ on the outer surface of the mockup, as discussed above. The constant of integration $C_{2}=T_{o \text {, wall }}$ is directly evaluated from the boundary conditions $T_{(x-0)}=T_{o \text { wall }}$, which are known from the IR-thermography measurement. Consequently the temperature distribution is given by equation (6) .

$$
T(x)=-\frac{q_{v o l} \cdot x^{2}}{2 \cdot k}+T_{\text {owall }}
$$

Inserting $\boldsymbol{x}=\delta$ into equation (6), the temperature $T_{(x \quad \delta)}=T_{i \text { wall }}$ on the inner surface of the wall is given by equation (7).

$$
T_{\text {iwall }}=-\frac{q_{\text {vol }} \cdot \delta^{2}}{2 \cdot k}+T_{\text {owall }}=-\frac{q^{*} \cdot \delta}{2 \cdot k}+T_{\text {owall }}
$$

where $q^{*}=\delta \cdot q_{v o l}$ is the local power density, other symbols see equation (4) and text.

The desired value for $\Delta T_{\text {wall }}=T_{o \text { wall }}-T_{\text {t.wall }}$ is then given by equation (8) .

$$
\Delta T_{\text {wall }}=T_{\text {owall }}-T_{\text {t.wall }}=\frac{q^{*} \cdot \delta}{2 \cdot k}
$$

We see that $\Delta T_{\text {wall }}$ depends linearly on the local power density. In conclusion equation (7) shows that IR-thermography is an effective tool for the investigation of SEH experiments when knowledge of the inner surface temperature is needed.

The above considerations have another important consequence for the energy balance equation (9), on the inside surface. According to Figure 5 the condition $q^{*}{ }_{\text {cond }}=q^{*}{ }_{\text {conv }}$ has to be fulfilled. From equation (6) and by substituting $q^{*}=\delta \cdot q_{v o l}$ one obtains:

$$
\begin{gathered}
q_{\text {cond }}=k \cdot \frac{d T(x)}{d x \quad(x=\delta)}^{=} q_{v o l} \cdot \delta=q^{*}=q_{c o n v}=\frac{T_{\text {lwall }}-T_{\text {bulk }}}{h} \\
q^{*}=\frac{T_{\text {l.wall }}-T_{\text {hulk }}}{h}
\end{gathered}
$$

where $h$ is local thermal resistivity, $k$ - thermal conductivity, $q^{*}$ - local convective heat flux, $T_{t \text { wall }}$ - temperature on the inner surface of the window wall, $T_{\text {bulk }}$ - mercury bulk temperature. 
Substitution of the temperature difference expression (10)

$$
\Delta T_{i \text { bulk }}=T_{i \text { wall }}-T_{\text {bulk }}
$$

into equation (9) gives equation (11).

$$
q^{*}=\frac{T_{i \text { wall }}-T_{\text {bulk }}}{h}=\frac{\Delta T_{i . b u l k}}{h}
$$

Equation (11) in cases where the local heat flux $q^{*}$ on the inner surface of the wall is known, enables the local thermal resistivity $h$ to be calculated according to formula (12).

$$
h=\frac{T_{i . w a l l}-T_{\text {bulk }}}{q^{*}}=\frac{\Delta T_{i . b u l k}}{q^{*}}
$$

If for compared cases two values of the local heat flux $q^{*}$ are unknown but equal, the configuration with the smaller $\Delta T_{i . b u l k}$ corresponds to the better cooled design. Thus equation (10) can be used for relative comparisons between investigated configurations.

As IR thermography allows only the temperature of the outer surface of the window to be measured, we are led to define another similar temperature difference expression as denoted in equation (13) .

$$
\Delta T_{\text {obulk }}=T_{\text {owall }}-T_{\text {bulk }}
$$

where $\boldsymbol{T}_{\text {o.wall }}$ is temperature on the outer surface of the window wall, - all another symbols as before used.

By substitution of equation (7) into equation (12) and with the help of equation (13) one obtains:

$$
h=\frac{T_{i . w a l l}-T_{\text {bulk }}}{q^{*}}=\frac{T_{\text {owall }}-\frac{q^{*} \cdot \delta}{2 k}-T_{\text {bulk }}}{q^{*}}=\frac{\Delta T_{\text {obulk }}}{q^{*}}-\frac{\delta}{2 k}
$$

The comparison of equation (12) with equation (14) shows, that for an exact determination of cooling efficiency by the IR thermography method, the value of the local thermal resistivity $h$ obtained from a measurement of the outer surface temperature must be amended by the subtraction of a constant term $\delta / 2 k$.

Notwithstanding this small modification, it remains true, that for constant local heat flux $q^{*}$, configurations with smaller values of $\Delta T_{\text {o.bulk }}$ corresponds to better cooled designs, so that equation (13) can be used to compare the various cooling configurations with one another.

\subsection{Visualization and comparison of relative cooling efficiencies}

Thanks to the calibration pre-tests and the simultaneous use of IR thermography and thermocouples, the measurement of the absolute temperature of the surface of the mockup achieved the good accuracy of $+/-0.5^{\circ} \mathrm{C}$. Use of 12-bit digital resolution (4096 levels) for the relatively small temperature range (from $-10^{\circ} \mathrm{C}$ to $+80^{\circ} \mathrm{C}$ ) allowed even very small $\left(0.1^{\circ} \mathrm{C}\right.$ ) temperature differences to be registered. The expectation that the dynamic behavior of these very small changes could be made apparent with a time resolution of $20 \mathrm{~Hz}$, was fulfilled. The high sensitivity obtained is in particular due to the use of volumetric heat generation and has helped to obtain astonishingly good visualization of the flow pattern from 2-D thermograms measured on the outside surface of the window.

As it was mentioned in the subsection 4.6 the effectiveness of the cooling under different flow conditions can be assessed by looking at the local thermal resistivity $h$ (equations (12) or (14); the smaller the thermal resistivity, the better the cooling at this point.

Likewise as it was previously discussed in the subsection 4.6. , when the convective local heat flux $q^{*}$ is unknown but constant for several compared cases (e.g. with different cooling configurations), the smaller $\Delta T_{\text {i.bulk }}$ (equation (10) ) or $\Delta T_{\text {o.bulk }}$ (equation (13) ) allows one to choose the preferred cooling conditions.

With the help of the emissivity charts, a precise correction was applied to each measurement pixel of all IR-thermograms recorded in the experiment sequences. After that the new difference thermograms or thermogram sequences were constructed according equation (13), thus allowing the best cooling configuration to be identified.

For example, the difference-thermograms $\Delta T_{\text {o.bulk }}$ of Figure 6 constructed according to equation (13), enable a comparison to be made between the natural convection case (buoyancy force driven only, Figure 6.a), and the forced convection case (buoyancy plus main stream pump flow rate $v=1.2 \mathrm{l} / \mathrm{s}$, Figure 6.b). All other conditions, i.e. the distribution of the heat flux density $q^{*}$ and the flow guide pipes with skew gap $=2 \mathrm{~cm}$, are the same in both experiments. With the IR-scanner in an axial position (see Figure 2), the axial projection of the hemispherical mockup leads to circular thermograms, as shown in Figure 6. The narrowest gap $(0.5 \mathrm{~cm})$ between the skew guiding pipe and the window wall is at 
the top of the thermograms, and the widest $3.5 \mathrm{~cm}$ gap at the bottom. The raw measurement data of the thermograms of Figure 6.a and 6.b were both taken in sequences of $20 \mathrm{~Hz}$ and later reduced to a $1 \mathrm{~Hz}$ sequence by averaging, emissivity correction and subtraction. On the internet page [http://wwwl.psi.ch/www_gfa_hn/asq/projects/liquid/liquid.html] this sequence is shown "live" and it is possible to observe the character of the flow and the efficiency of the heat transfer. In this animation the fast motion effect is used ( $1 \mathrm{~Hz}$-thermograms are replayed at 15 frames per second), which allows one to recognize the character of the flow more easily.

The thermographic $\Delta T_{\text {o.bulk }}$-visualization of the cooling shows the characteristic pattern of the streaming mercury quite well. In the case with natural convection only, the pattern is irregular, unstable and slower than in the case of forced convection, where the streaming mercury builds a very characteristic and stable "butterfly" pattern for the temperature field. The formation of this pattern is caused from small changes of temperature result in a so called "tracing" effect and allow the visualization of the mercury flow inside with astonishingly good resolution. When the heating was switched on, the flow of streaming mercury could be observed in fast motion.
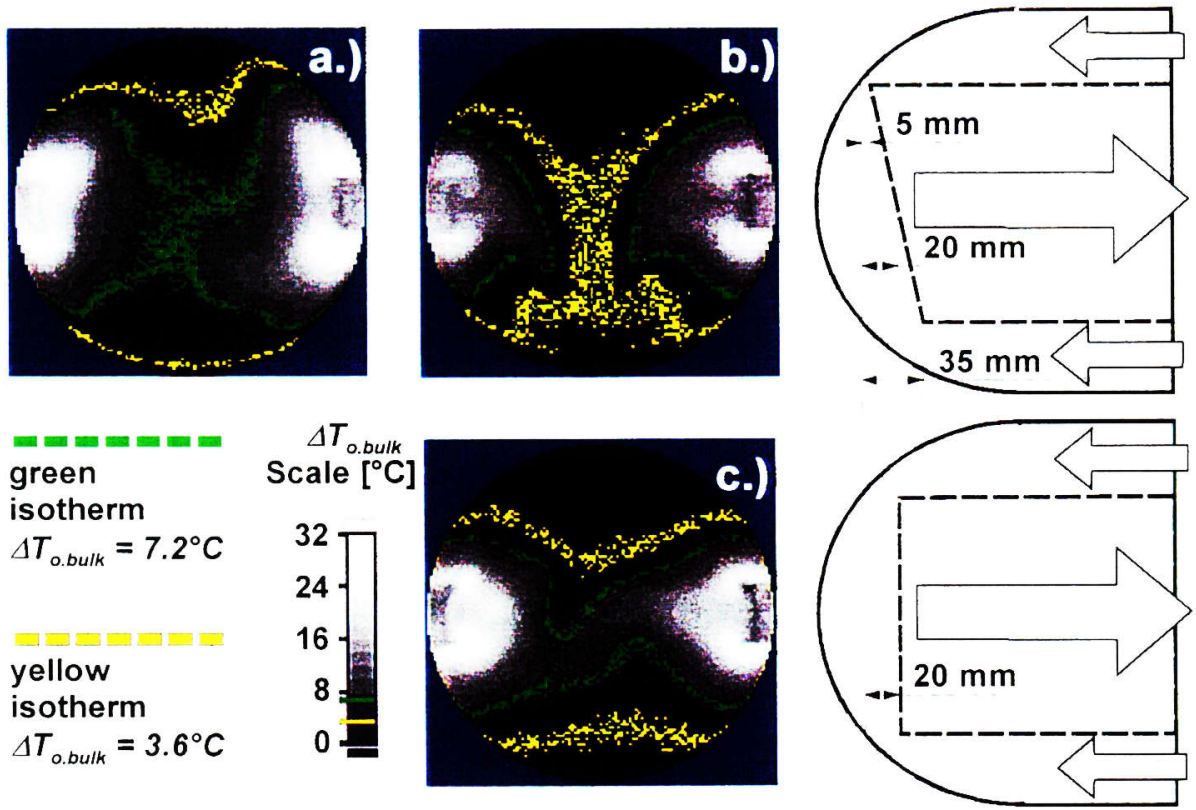

Figure 6. The $\Delta T_{\text {o.bulk }}$-visualization of the cooling. a.) The case with natural convection (without forced flow) and $2 \mathrm{~cm}$ skew gap for the guide pipe. b.) The case with forced convection (pump flow rate $1.2 \mathrm{l} / \mathrm{s}$ ) and $2 \mathrm{~cm}$ skew gap for the guide pipe. c.) The case with forced convection (pump flow $1.2 \mathrm{l} / \mathrm{s}$ ) and $2 \mathrm{~cm}$ flat gap of guide pipe.

As discussed above, the $\Delta T_{\text {o.bulk }}$ visualization shows more than the phenomenology alone. It is possible to obtain some important quantitative information. For example the comparison between natural and forced convection for a skew gap of $2 \mathrm{~cm}$, as presented here, shows that at the center of the window the forced convection cooling is better. The isothermal lines shown in Figure 6.a and 6.b, yellow for $\Delta T_{\text {o.bulk }}=3.6^{\circ} \mathrm{C}$ and green for $\Delta T_{\text {o.bulk }}=7.2^{\circ} \mathrm{C}$, immediately enable one to calculate that the thermal resistivity of forced convection with pump flow rate of $1.2 \mathrm{l} / \mathrm{s}$ is two times smaller i.e. better.

The next thermogram, Figure 6.c shows the IR-thermography $\Delta T_{\text {o.bulk }}$-visualization for another experiment. In this case the flow guide pipe had a flat gap of $2 \mathrm{~cm}$ and the same conditions as before were in effect for the heating and for the forced convection with a flow rate of $1.2 \mathrm{l} / \mathrm{s}$ in the main stream. For this experiment also, the animated $\Delta T_{\text {obulk }}$ sequence is available on the internet page [http:/wwwl.psi.ch/www_gfa_hn/asq/projects/liquid/liquid.html]. From the phenomenological point of view, it is very interesting to observe the development of the left twisting whirl (upturn flow against the force of gravity). The vortex shows the typical cooling effect where the middle point is worse cooled than the nearby points at larger radii.

Also in Figure 6.c, the green isotherm line for $\Delta T_{\text {o.bulk }}=7.2^{\circ} \mathrm{C}$ enables one to calculate, that the thermal resistivity for this case (forced convection of $1.2 \mathrm{l} / \mathrm{s}$ and $2 \mathrm{~cm}$ flat gap) is approximately two times greater, i.e. worse, than the case with the same main stream pump conditions $(1.2 \mathrm{l} / \mathrm{s})$ and a skew gap of $2 \mathrm{~cm}$, Figure 6.b. This of course assumes, as mentioned before, that the same conditions for the local heat flux density are in effect in both cases. 


\subsection{Estimation of the local power density distribution for the SEH-experiments}

The second part of goal \#2 from section 2 is to estimate and visualize the local thermal resistivity. In order to obtain the values of local thermal resistivity $h$ according to equation (14) a knowledge of the distribution of the local heat flux $q^{*}$ during the SEH experiments is indispensable. As can be seen on the thermogram of Figure 7, the distribution of the heat flux $q^{*}$ obtained with "Skin Effect" Heating is highly inhomogenous. The concentration of power density in the vicinity of both electrodes is obviously higher than in the central region of the mockup.
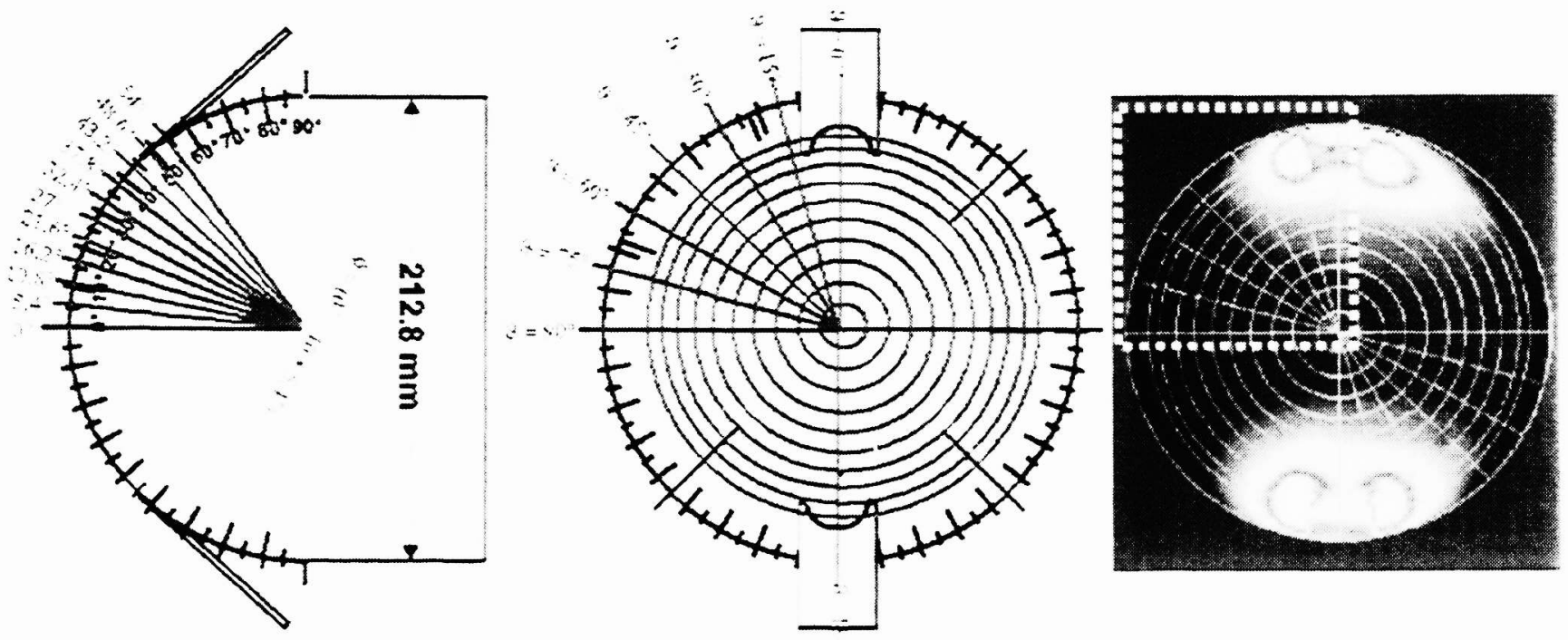

Figure 7. Estimation of the local power density $q_{\varphi .9}^{*}$. Discretization mesh for the measurement of the electrical potential drop.

Additional measurements for the estimation of the power density distribution were performed in IPUL -Riga for similar conditions as in the SEH experiments ${ }^{8 .}$. On one quarter of the surface of the mockup (assumed to have two axes of symmetry), the electrical potential was measured in $7 l$ node points of a $\varphi-\vartheta$ mesh (see Figure 7). During these experiments and for security reasons the SEH heating was done with an electric current $I_{A}=2025 \mathrm{~A}$, different from the value $I_{B}$ $2570 \mathrm{~A}$, as used in the IR thermography measurements.

The 2-D distribution of power density, see equation (15), was calculated in the middle points of the discrete elements. According to resistance heating (Ohmic law) we have:

$$
q_{\varphi, 9}^{*}=\left[\left(\frac{\Delta U_{\varphi}}{\Delta L_{\varphi}}\right)^{2}+\left(\frac{\Delta U_{\vartheta}}{\Delta L_{\vartheta}}\right)^{2}\right] \cdot \delta \cdot \sigma
$$

where: $q_{\varphi . g}^{*}$ is a local power density in the middle of discrete element, $\delta$ - depth of penetration according to equation (1). $\sigma$ - electric conductivity, $\Delta U \varphi$ - potential drop in middle point of discrete element in direction $\varphi, A U \vartheta$ - potential drop in middle point of discrete element in direction $\vartheta, A L \varphi$ - length of elements in direction $\varphi ;(A L \varphi-c o n s t=R A \varphi \Pi / 180$ $10 \mathrm{~mm}), \Delta L \vartheta$ - length of elements in direction $9:(\Delta L Y=R \sin \varphi \Delta 9 \Pi / 180), \quad R$ - radius of hemispherical mockup : $(R$ $106.37 \mathrm{~mm}), \Delta \varphi$-discretization angle in direction $\varphi ;\left(\Delta \varphi=5.4^{\circ}\right), \Delta \vartheta$ - discretization angle in direction $\vartheta ;\left(\Lambda \vartheta=15^{\circ}\right)$

The scaling to the local power density $q_{\varphi .9}^{*}$ deposited by a current of $2570 \mathrm{~A}$, as used during the experiments, was done with formula (16).

$$
q_{\varphi, 9}^{*, B}=q_{\varphi, 9}^{* A} \cdot\left(\frac{I_{B}}{I_{A}}\right)^{2}
$$

For these time-consuming measurements a simple hand probe was used to measure single points one after the other. Unfortunately, and contrary to expectations, the measurements showed that the heating is insufficiently stable. Nevertheless every effort was made to derive from these potential drop measurements a realistic value for the characteristic distri- 
bution of power density ${ }^{4 .)}$. Finally after consideration of the accuracy of the measurements, it must be concluded, that this edition of SEH heating had too many uncertainties to allow a precise estimation of the local thermal resistivity $h$. Consequently we have to use an improved heating with a precise knowing of heat flux distribution in the future ${ }^{4 .)}$.

\section{CONCLUSIONS}

With respect to goals \#1 and \#2 of section 2, the so called 2DD method (with using of IR-thermography in SEH experiments, in conjunction with the available time- space- and temperature-resolution equipment of AGEMA THV900), on the one hand achieves satisfactory results for :

1. The visualization of the mercury flow behind the $2.88 \mathrm{~mm}$ thick steel wall of the mockup of the proton beam entry window of SINQ.

2. The comparison of the relative local thermal resistivity $h$ (or the local convective heat transfer coefficient $\alpha$ ) between different flow or cooling configurations in the quasi steady-state condition. This conclusion does not require knowledge of the absolute value of the local heat flux $q^{*}$, but it is indispensable to make sure that:

- The distribution of the local heat flux $q^{*}$ for all compared configurations remains the same.

- The mercury bulk temperature is simultaneously controlled.

On the other hand the 2DD method was not yet sufficiently precise for:

3. The determination of the local thermal resistivity $h$, because of uncertainties in the estimation of the local heat flux $q^{*}$ resulting from the current edition of the SEH heating. The efforts to develop a system of heating allowing the local heat flux $q^{*}$ to be determined with sufficient precision will be continued.

\section{ACKNOWLEDGEMENTS}

This work was partially supported by the European Spallation Source (ESS) Project, Forschungszentrum Jülich GmbH, FZJ, D-52425 Jülich, Germany.

\section{REFERENCES}

1.) G.S. Bauer, "Technology issues in the design of medium-to-high power spallation targets for accelerator driven systems", J. Phys. IV France, Vol. 9, pp. Pr7-91-Pr7-113, 1999.

2.) Y. Takeda, H. Kikura, and G.S. Bauer, "Flow Measurement in a SINQ Mockup Target Using Mercury", Proceedings of the $14^{\text {th }}$ Meeting of the International Collaboration on Advanced Neutron Sources, (ICANS XIV), pp.321-327, Starved Rock Lodge, Argonne National Laboratory, 1998.

3.) I. Platnieks, G.S. Bauer, O. Lielausis, and Y. Takeda, "Measurements of Heat Transfer at the Beam Window in a Mockup Target for SINQ Using Mercury", Proceedings of the $14^{\text {th }}$ Meeting of the International Collaboration on Advanced Neutron Sources, (ICANS XIV), pp.382-395, Starved Rock Lodge, Argonne National Laboratory,1998.

4.) J.A. Patorski, Y. Takeda, "Liquid Metal Target; Experimental Study (Phase 1) of the Cooling of a SINQ-type Mercury Target Window", to be published in European Spallation Source Report, Vol. ESS 00-101-T, April/May 2000, Forschungszentrum Jülich GmbH, FZJ, D-52425 Jülich, Germany.

5.) AGEMA Infrared Sytems, "Thermovision ${ }^{\circledR} 900$ Series User's Manual" AGEMA Infrared Sytems Publication No.556960, 1993.

6.) S. Dementjew, "Pre-test for Skin Effect Heating", Correspondence from IPUL to PSI, February 1998.

7.) M.F. Modest, Radiative Heat Transfer, Ch.3, McGraw-Hill, Inc., New York, 1993.

8.) S. Dementjew, "Measurement of the distribution of the local heat flux during the SEH experiments ", Correspondence from IPUL to PSI, August 1998. 